\title{
Nation-scale primary prevention to reduce newly incident adolescent drug use: The issue of lag time
}

\author{
Hui Cheng ${ }^{\text {Corresp., }}{ }^{1}$, Dukernse Augustin ${ }^{1,2}$ ， Eric H Glass ${ }^{2}$ ， James C. Anthony ${ }^{\text {Corresp. } 1}$ \\ 1 Department of Epidemiology \& Biostatistics, Michigan State University, East Lansing, Michigan, United States \\ 2 Michigan State University, East Lansing, Michigan, United States \\ Corresponding Authors: Hui Cheng, James C. Anthony \\ Email address: chenghu2@msu.edu, janthony@msu.edu
}

Background: There is limited evidence about the effects of United States (US) nation-level policy changes on the incidence of alcohol drinking and tobacco smoking. To investigate the potential primary prevention effects on precocious drug use and to clarify lag-time issues, we estimated incidence rates for specified intervals anticipating and lagging after drug policy enactment. Our hypotheses are a) reductions in underage drinking or smoking onset and b) increases of incidence at the legal age (i.e., 21 for drinking and 18 for smoking).

Methods: The study population is 12-to-21-year-old non-institutionalized US civilian residents. Estimates are from 30 community samples drawn to be nationally representative for the US National Surveys on Drug Use and Health 1979-2015. Estimates were year-by-year annual incidence rates for alcohol drinking and tobacco smoking by 12-to-21-year-olds, age by age. Meta-regressions estimate age-specific incidence over time.

Results: Incidence of underage alcohol drinking declined and followed a trend line that started before 1984 enactment of the National Minimum Drinking Age Act, but increased drinking incidence for 21 year olds was observed approximately 10 years after policy enactment. Eight years after Synar amendment enactment, evidence of reduced smoking incidence started to emerge. Among 18 year olds, a slight increase in tobacco smoking incidence occurred about 10 years after the Synar amendment.

Conclusion: Once nation-level policies affecting drug sales to minors are enacted, one might have to wait almost a decade before seeing tangible policy effects on drug use incidence rates. 
November 26, 2018

3

Title: Nation-Scale Primary Prevention to Reduce Newly Incident Adolescent Drug Use:

The Issue of Lag Time

6 Authors: Hui G. Cheng, Dukernse Augustin, Eric H. Glass, and James C. Anthony

7 Department of Epidemiology and Biostatistics, Michigan State University, 909 Fee Rd. Room

8 B601, East Lansing, 48824, USA

9 Authors email:

10 Hui Cheng: chengyaojin@yahoo.com

11 Dukernse Augustin: august51@,msu.edu

12 Eric H. Glass: glasser3@msu.edu

13 James C. Anthony: janthony@msu.edu

14

15 Correspondence:

16 Dr. James C. Anthony (janthony@msu.edu) or Dr. Hui Cheng (chengyaojin@yahoo.com)

17909 Fee Rd. Room B601

18 Michigan State University

19 East Lansing, 48824, USA 


\section{Abstract}

22 Background: There is limited evidence about the effects of United States (US) nation-level policy changes on the incidence of alcohol drinking and tobacco smoking. To investigate the potential primary prevention effects on precocious drug use and to clarify lag-time issues, we estimated incidence rates for specified intervals anticipating and lagging after drug policy enactment. Our hypotheses are a) reductions in underage drinking or smoking onset and b) increases of incidence at the legal age (i.e., 21 for drinking and 18 for smoking).

Methods: The study population is 12-to-21-year-old non-institutionalized US civilian residents.

Estimates are from 30 community samples drawn to be nationally representative for the US National Surveys on Drug Use and Health 1979-2015. Estimates were year-by-year annual incidence rates for alcohol drinking and tobacco smoking by 12-to-21-year-olds, age by age. Meta-regressions estimate age-specific incidence over time.

Results: Incidence of underage alcohol drinking declined and followed a trend line that started before 1984 enactment of the National Minimum Drinking Age Act, but increased drinking incidence for 21 year olds was observed approximately 10 years after policy enactment. Eight years after Synar amendment enactment, evidence of reduced smoking incidence started to emerge. Among 18 year olds, a slight increase in tobacco smoking incidence occurred about 10 years after the Synar amendment.

Conclusion: Once nation-level policies affecting drug sales to minors are enacted, one might have to wait almost a decade before seeing tangible policy effects on drug use incidence rates. 
42

43

44

\section{Introduction}

A leading cause of disease burden in young people is precocious and underage use of alcohol, tobacco, and other drugs, by which we mean starting to use one or more of these drugs extramedically before age 18 years or age 21 years (Hall et al., 2016; Lim et al., 2012). In the United States (US), a series of nation-level policies has been put into place to help shape primary prevention of these forms of precocious drug use, and to deter new onsets of underage drug use. In the case of alcohol, the 1984 National Minimum Drinking Age Act (NMDAA) required all states to ban sales of alcoholic beverages to individuals below age 21 years, and to do so during or before December 31, 1986; otherwise, the state would lose a portion of its federal highway funds (Toomey, Nelson, \& Lenk, 2009). In the case of tobacco (DiFranza \& Dussault, 2005), the 1992 Synar Amendment prohibited sales or distribution of tobacco products to individuals before the $18^{\text {th }}$ birthday. Otherwise, the state would not receive the full award of the federal Substance Abuse Prevention and Treatment Block Grant (https://www.samhsa.gov/synar/about, accessed January 13, 2017).

Faced with challenges of a true experimental design, a series of studies applied novel statistical models to observational data to infer the potential causal role of these policies in alcohol drinking and smoking behaviors (e.g., regression discontinuity and instrumental variable approaches). These studies have consistently documented greater alcohol consumption among 21 year olds compared to 20 year olds, lending support to the idea that minimum legal drinking age can shaping drinking behaviors (Carpenter \& Dobkin, 2009; Yörük \& Yörük, 2013). The evidence about smoking behavior is less clear (Ertan Yörük \& Yörük, 2016). With a focus on variations in the population-level incidence rates for alcohol drinking (i.e., the probability of 
65 becoming a user) over multiple years, in our own work, we have added new evidence about the

66 peak risk of drinking onset at 21 years of age in the contemporary US. This published evidence is

67 quite consistent with the notion that the minimum legal drinking age plays a role in shaping

68 drinking onset as young people transition from late adolescence to age 21 years (Cheng, Cantave,

69 \& Anthony, 2016b, 2016a; Cheng, Lopez-Quintero, \& Anthony, 2017).

71 Nonetheless, a policy-maker might ask about how quickly a regulatory change in minimum legal age for any given drug would be followed by tangible reductions in population-level incidence rate estimates driven by the individual-level probability of starting to use that drug. This epidemiological question about lag time from policy enactment until an observable policypreventive effect on population-level drug use incidence rates generally has been mentioned in discussions about methods (Pacula, Kilmer, Wagenaar, Chaloupka, \& Caulkins, 2014). Indeed, many of the current evaluations of cannabis policy change are based on assumptions about relatively short lag times, and the mixed evidence in the extensive drug policy evaluation literature reflects, to some degree, an expectation of fairly rapid effects of nation-level policies (e.g., Fichtenberg \& Glantz, 2002; Holford, Levy, et al., 2014; Wagenaar \& Toomey, 2002). Virtually all of these studies specified a lag time interval at values of 1-5 years. Hence, we were prompted to wonder whether the lag time specification of 1-5 years might be too short when the goal is to see a policy-preventive effect on population-level incidence rates. We speculated that a lag time of ten years or greater might qualify as a more realistic working hypothesis when the context is that of a relatively large and heterogeneous country such as the United States, and when states might launch initial policy implementation soon after policy enactment, but might not achieve steady state in policy implementation until many years after policy enactment. 
89 Another important issue is confronted when these policy analyses are evaluated from an

90

91

92

93

94

epidemiological perspective that draws a crisp distinction between prevalence estimates (the probability of being a user) versus incidence estimates (the probability of becoming a user). In this context, we note that 'primary prevention' of drug policies, by definition, should be seeking a reduction in the incidence rate (formation of newly incident users), and might not have any direct secondary prevention effect of shortening duration. Moreover, the prevalence of active drug use is determined in part by carry-forward of drug use that started in prior years and persists into each subsequent year. The incidence rate has no such carry-forward of long-duration drug use. Incidence pertains to the rate at which newly incident drug users are added to the user population, conditional on no prior use. On the basis of general epidemiological principles, we can formulate a working hypothesis that the primary prevention impact of a nation-level policy change, if any, might be seen first in the incidence rate. If nation-level policy affects prevalence but not incidence, this result signals possible policy disruption of on-going drug use, with no primary prevention effects.

With these thoughts in mind, we decided to take an initial empirical step in an epidemiological investigation of the incidence rate evidence about two separate United States (US) nation-level policy changes clearly intended to have beneficial primary prevention effects on precocious or otherwise underage drug use: (1) the NMDAA, and (2) the Synar, as described above. For this initial step, we have produced year-by-year estimates for the annual incidence rates for drug use experiences of 12-to-21-year-olds, age by age, before and after enactment of these two policies. Our working hypothesis, specified prior to data analysis, was that any primary prevention effect 
111 of these nation-level policies on incidence rates might not be seen within a lag time of five years.

112 A 10-year lag time might be required.

113

114 In order to look into our working hypothesis that it might take 10 years or more to see drug

115 policy impact on adolescent incidence rates, we constructed a US time series of incidence rate

116 estimates for the years before and after the US enacted these two drug policies. A secondary

117 hypothesized change is an increase of incidence at the legal age (i.e., 21 for drinking and 18 for

118 smoking), which is an empirically grounded hypothesis based on some recent work on age-

119 specific incidence rates for starting to drink alcoholic beverages (Cheng et al., 2016a). This

120 empirical research report describes what we found.

121

122

2. Materials and Methods

\subsection{Study population and sample}

124 The study population is non-institutionalized civilian residents aged 12 years and above living in

125 the US, sampled from 30 national surveys conducted by the US federal government. From 1979

126 to 2001, these surveys were named National Household Survey on Drug Abuse (NHSDA),

127 which changed to National Survey on Drug Use and Health (NSDUH) in 2002 (United States.

128 2014). In all surveys, multi-stage area probability sampling was used to yield US nationally

129 representative samples (United States, 2012). More details about sampling procedures and

130 demographic characteristics of the NHSDA and NSDUH surveys are provided on the NSDUH

131 website (https://www.datafiles.samhsa.gov/study-series/national-survey-drug-use-and-health-

132 nsduh-nid13517, accessed November 10, 2018). All NSDUH participants were recruited via 
133 child assent and parental or adult consent, based upon protocols approved by cognizant human

134 subjects protection committees.

135

136 From 1979 to 1990, the sample size varied from 5000 to 10,000; between 1991 and 1998, the

137 sample sizes were between 18,000 and 30,000; since 1999, the sample size has been $\sim 55,000$

138 each year (United States, 2012). For this analysis, we focused on the 'at risk' population for the

139 onset of alcohol drinking or cigarette smoking. Supplementary Table S2 and S3 provides year-

140 and age-specific sample sizes of the 'at risk' populations. The aggregated sample sizes for this

141 study are 358,205 for alcohol drinking and 429,348 for tobacco smoking (i.e., denominators for

142 incidence estimates).

\subsection{Assessment and measures}

During NSDUH fieldwork, participants completed interviews, with standardized multi-item modules on alcohol, tobacco, and other health topics. These items assessed the participant's age when they had their first drink or smoked their first cigarette, as well as recency of use, and

148 lifetime history of use. Minor changes in wording occurred during the 37 years of study interval.

149 Supplementary Table 1 provides more details about changes in survey questions as well as 150 changes in methodology that are most relevant to this analysis (United States. 2014). In this 151 study, newly incident users are individuals whose onset was at the current age or a year younger.

152 This approach yields valid estimates for annual incidence and constrains memory errors 153 (Barondess, Meyer, Boinapally, Fairman, \& Anthony, 2010; Kuntsche, Rossow, Engels, \&

154 Kuntsche, 2016; Shillington, Woodruff, Clapp, Reed, \& Lemus, 2012). Age is based on the date 155 of birth. Information from the survey roster was drawn when the question was skipped. 


\section{$157 \quad 2.3$ Analysis approach}

158 In this study, incidence was conceptualized as the number of newly incident users who started

159 drinking or smoking at a specific age rising from the 'at risk' population comprised of never

160 users assessed at a specific age and newly incident users. A previous study provides more details

161 about this approach for the estimation of age-specific incidence (Cheng et al., 2017). From 1999

162 and onward, the participants' age is only available in aggregated groups for those older than 21

163 years in the public downloadable datasets. For example, 22-23 year olds were grouped together,

164 and 24-25 year olds were grouped together. To accommodate this change, newly incident

165 drinkers or smokers at 21 included those who started drinking or smoking at 21 and assessed at

16621 and half of those who started drinking or smoking at 21 and assessed at 22 or 23; annual

167 incidence was estimated for 22-23 as a group (instead of for 22 year olds and 23 year olds

168 separately). The incidence estimate was operationalized as analysis-weighted numbers of newly

169 incident users divided by analysis-weighted numbers of those at risk to start using. In subsequent

170 steps, we plotted the estimated annual incidence for visualization of change over time.

171

172 Next, we used meta-regression to compare incidence estimates between four time periods based

173 on the NMDAA and Synar amendments (Thompson \& Sharp, 1999). Conceptually, meta-

174 regression aggregates evidence to address sources of heterogeneity in related estimates from

175 multiple studies on the same topic. with each study viewed as a non-exact replicate based on

176 different study populations across various spatio-temporal locations (Borenstein, Hedges,

177 Higgins, \& Rothstein, 2009). Meta-regression takes into account sampling variability (via error

178 terms) and study-specific deviations from the general mean (via random effect terms) in order to 
179 quantify estimated heterogeneity in estimates associated with study-specific characteristics (e.g.,

180 study period). In this research, the annual NSDUH surveys over 37 years are the 'multiple

181 studies,' each with an independent replicate sample of the US study population as it changed

182 year by year, and with methods changes as noted in Supplementary Table S1, and the estimands

183 are incidence rate estimates. One might use other analysis approaches, but our pre-specification

184 for the analysis protocol was to make use of meta-regressions and to report what we learned from 185 meta-regressions. Our data sharing plan makes it possible for others to use different approaches

186 and to learn whether different approaches might yield different results. For alcohol drinking, the

187 four periods are 1979-1986 (pre-NMDAA, Period 0), 1987-1996 (immediate post-NMDAA,

188 Period 1), 1997-2006 (post-NMDAA, Period 2), and 2007-2015 (post-post-NMDAA, Period 3).

189 For tobacco smoking, the four periods are 1979-1992 (pre-Synar, Period 0), 1993-1999

190 (immediate post-Synar, Period 1), 2000-2007 (post-Synar, Period 2), and 2008-2015 (post-post-

191 Synar, Period 3).

192 Analysis weights account for sample selection probabilities and post-stratification adjustment

193 factors that yield US Census subpopulation counts.

\section{Result}

Figure 1 depicts a visualization of change over time for each age stratum. Supplement Table S3

197 shows estimated age-specific annual incidence of drinking among 12-23 year olds, year by year, 198 from 1979 to 2015. Among adolescents 12-17 years of age, sharp declines occurred before 199 NMDAA and continued into the early 1990s, paused during the 1990s, and a second wave of 200 declines with smaller magnitude occurred afterward. Consistent with our hypothesis, annual 201 incidence of drinking among 21 year olds rose; however, the increase was not observed until 
2021991 (i.e., seven years after NMDAA). Among 18-20 year olds, the largest decrease occurred 203 before the NMDAA (i.e., between 1979 and 1982). The annual incidence of drinking remained

204 fairly stable at $40 \%$ for 18 year olds, whereas the decline continued for 19 -and-20-year-olds 205 during the 10 years after NMDAA (i.e., 1985 to 1994). Since 1998, drinking incidence has been 206 fairly stable among 18-20 year olds. In 22-23 year olds, annual incidence of alcohol drinking 207 remained at a low level (i.e., $\leq 10 \%$ except for 1979).

208

As shown in Figure 2 and Supplement Table S4, there was a universal rise in the annual incidence of tobacco cigarette smoking immediately after the Synar amendment in all age groups except for 22-23 year olds between 1993 and 1998, followed by a declining trend that persisted to the most recent survey year among adolescents. Sharper declines were seen for early adolescents compared to later adolescents. The decline occurred earlier among 12 year olds in 1995. Among 18 year olds, no decline was observed. Instead, an ascendance is seen between 2005 and 2010 (i.e., 13 years after Synar). Among 19-23 year olds, the incidence of tobacco cigarette smoking fluctuated without any clear time trend since the late $90 \mathrm{~s}$.

Findings from meta-regression disclosed similar patterns as described above (Table 1). Major drops among 12-20 year olds occurred during the first post-NMDAA period, stopped during the second decade after NMDAA, and resumed with a lesser magnitude during the third decade after NMDAA. Increases among 21 year olds occurred in the second decade and continued into the third decade after NMDAA. 
224 For tobacco smoking, the first seven years after the Synar amendment saw universal increase in 225 smoking incidence among all age groups except for 12 and 13 year olds (Supplementary Table 226 S4). Compared to the first post-Synar period (i.e., 1993-1999), tobacco smoking incidence

227 during the second post-Synar interval declined for all adolescents and persisted to the most 228 recent period under study. In contrast, incidence of tobacco smoking increased slightly among 18 229 year olds, and remained stable for 19-23 year olds during the second and third post-Synar 230 periods.

Readers may be interested in assessing the shift of age patterns over time. Supplementary Figure 1 presents age-specific patterns for alcohol drinking incidence. Before 1986, drinking incidence was high among 15 to 20 year olds. From 1988 to 1994, the peak was generally observed between 16 and 18, and a secondary peak at 21 gradually emerged. From 1995 and onward, the peak at 21 became prominent and the incidence curve shifted from a bi-modular shape to a unimodular shape. A noticeable dip at 19 and 20 years was seen in 1993 for the first time and became consistent since 1998 .

Supplementary Figure 2 shows the age pattern for tobacco cigarette smoking year by year. 
248 The main findings of this study may be summarized succinctly. First, First, declines in incidence 249 of adolescent alcohol drinking after NMDAA is a continuation of a decreasing trend that started

250 before the NMDAA. The rise among 21 year olds did not emerge until approximately 10 years

251 after NMDAA. Two decades after NMDAA, a second decline occurred among adolescents.

252 Second, the annual incidence of tobacco cigarette smoking rose immediately after the Synar

253 amendment in all age groups. Declines in adolescent tobacco smoking incidence occurred at least

254 eight years after the Synar amendment and persisted until the most recent year under study.

255 Among 18 year olds, a slight increase in tobacco smoking incidence occurred about 10 years

256 after the Synar amendment and lasted for about 10 years. Third, the shift of age pattern in

257 incidence occurred gradually. Prominent onset peaks of autonomous drinking or smoking at the

258 legal age did not occur until approximately 10 years after the NMDAA or Synar.

Before detailed discussion of these results, several of the more important study limitations merit attention. Of central concern is the observational nature of the study which precludes definitive evidence for any causal relationships. Many changes related to alcohol drinking and tobacco smoking co-occurred with NMDAA and Synar. For example, there were numerous nation-, state, and local-level policies and movements, including excise taxes on tobacco and alcohol products, state-level MLDA or minimum legal tobacco purchasing age (MLTPA), mass media campaigns, and grassroots movements throughout the study period (Farrelly, Niederdeppe, \&

267 Yarsevich, 2003; Lum, Barnes, \& Glantz, 2009; Toomey et al., 2009), as well as variations in the 268 implementation of state-level policies over time (Nelson, Xuan, Blanchette, Heeren, \& Naimi, 
269 2015; Spivak \& Monnat, 2015). The observed changes are results of the national NMDAA and

270 Synar combined with these various factors.

271 We wish to return to the issue of unmeasured and uncontrolled variables in our meta-regressions.

272 We are hopeful that readers will appreciate that this is an observational study based on a

273 national-level surveillance of alcohol and tobacco use in the US population over a 37 year

274 interval. Nation-level surveillance of this type cannot cover all potentially influential variables

275 that might show variation within or across US jurisdictions during a 37-year interval, but should

276 not be subject to influence from relatively stable variables such as the ratio of males and females

277 among newborns in the US population. Nevertheless, there are some variables that have changed

278 during this 37-year interval and that might have a bearing on the estimation of lag times from the

279 year of policy enactment until a tangible reduction in drinking or smoking incidence rates can be

280 seen. We must leave the control of these variables to a future research project that can build from

281 our meta-regression findings, and in this set of variables a future research team will face some

282 special challenges that involve topics such as disposable income and local alcohol/tobacco taxes,

283 the smoking behavior of parents or siblings or peers or adult co-workers, and the relative balance

284 of US-born versus non-US-born residents. A participant's disposable income is not a variable

285 that is measured in these surveys, nor is the local area or state alcohol or tobacco tax level as it

286 has varied over time. A measure of parental or other environmental alcohol and tobacco

287 consumption can be created and introduced as a meta-regression covariate, but we have not done

288 so in this initial meta-regression work. The same is true for the relative balance of US-born

289 versus non-US-born residents of the US, given that the recent US population distributions show a

290 reduced proportion of US-born residents, relative to what was seen in the first 10 years of the 37-

291 year interval under study. We are not at all sure that changing variables of this type would have a 
292 major impact on the meta-regression estimates about lag time for policy effects on population-

293 level incidence rates, and we must leave these issues as ones that can be addressed in future

294 investigations that build from the present investigation.

295

296 There are several significant methodological changes during the 37 years under study. First, the

297 NSDUH sampling frame changed in 1991 to include Alaska and Hawaii, as well as civilians

298 living in non-institutional group quarters (United States. 2014). Nonetheless, neither NMDAA

299 nor Synar happened in 1991, and no out-of-line estimates were observed in 1991 for either

300 alcohol drinking or tobacco smoking. Second, the sample sizes of the earliest surveys are

301 relatively small which constrains the power of statistical inference. Third, the time span of the

302 current analysis is restricted to 1979 and later due to the availability of data. Therefore, we were

303 not able to assess trends in alcohol drinking for an extended pre-NMDAA period. Fourth, the

304 survey questions related to alcohol drinking and tobacco smoking changed several times. Of

305 special interest is the change of smoking question in 1994, which might have contributed to the

306 increase in tobacco incidence. Nonetheless, the increase is observed in other population surveys

307 for which survey questions did not change in 1994 (e.g., MTF and National Youth Risk Behavior

308 Survey; Johnston et al. 2014; United States 2013). Therefore, we do not consider the increase an

309 artifact wholly attributable to change in survey questions. In addition, the method of survey

310 administration changed from face-to-face interview to ACASI in 1999. Nonetheless, no

311 discernible change was observed around 1999. Although several method changes, including the

312 provision of a $\$ 30$ incentive, occurred in 2002 and the survey name changed, questions about the

313 onset of drinking and smoking as well as the sampling strategy remained the same. In addition, 
314 no substantial changes in estimated incidence of drinking or smoking was observed. Therefore,

315 we consider that these changes had minimum influence on our estimates for this study.

317 Although the assessment of first full drink is fairly straightforward, the first use of tobacco

318 product is less so. Other forms of tobacco products exist, including chewing tobacco, water pipe

319 smoking, and recently vaping (i.e., e-cigarettes). In this study, we only focused on the first

320 tobacco cigarette smoking in order to generate comparable estimates. (The use of other forms of

321 tobacco products was not assessed in earlier surveys.) According to the NSDUH data,

322 approximately $6 \%$ to $10 \%$ of all tobacco users had never smoked tobacco cigarettes between

3232002 and 2015. (NSDUH has not been assessing e-cigarette use.) Given the relatively small

324 proportions, we judge that the use of other tobacco product cannot fully account for the observed 325 changes over time.

327 Notwithstanding limitations such as these, the study findings are of interest because we provided an over-time view of age-specific changes in the onset of drinking and smoking using nationally

329 representative data with fairly consistent methodology. For this study, we specified the study population to include essentially all non-institutionalized civilian young people in the US, even if the young people had dropped out of school or had become disengaged from school, an experience that often signals excess incidence rates of drug use (e.g., Sweeten et al., 2009). In this fashion, we avoided potential study biases faced when nation-level policy has been evaluated based on school survey data. 
336 This study population feature of the research design might be especially important because

337 during the years under study there were concurrent initiatives to expel, suspend, or restrict school

338 attendance of students who had started to smoke tobacco or to drink alcoholic beverages

339 (DiFranza \& Dussault, 2005; Toomey et al., 2009). In consequence, corresponding incidence

340 rates for school-attending adolescents would not provide a complete picture of the

341 epidemiological phenomenon. In addition, the available school surveys generally have asked

342 about 'grade' of starting drug use, but not 'age 'of starting drug use, with 'grade' being undefined

343 for one who no longer attends school. The NMDAA and Synar policies focus on age of starting

344 to use, but not on grade of starting to use. Moreover, our intent was to reach out and to capture

345 the experience of 18-to-21-year-olds, many of whom are not included in the sampling frames for

346 US surveillance of school-attending youths, year by year, from the late 1970s through the 2000s.

348 In this study, we found large drops in adolescent drinking incidence before and after the

349 NMDAA. It is noteworthy that NMDAA is not the first to impose age 21 as the legal minimum

350 drinking age (LMDA). In 1933 (after the nation-wide prohibition was removed), a LMDA of 21

351 was enacted in all states until 1970. Between 1970 and 1975, 29 states lowered the LMDA to 18.

352 Between 1978 and 1986, these states reinstated the LMDA to 21 due to concerns about a rise of

353 motor vehicle accidents among 18-to-20-year-olds (Toomey et al., 2009). The implementation of

354 NMDAA happened during 1978 to 1986 in different states. Therefore, whether the decline in

355 adolescent drinking onset is at least partially due to NMDAA is not clear. Nonetheless, the

356 distinctive peak at 21 was not seen until some 10 years after NMDAA. 
358 For tobacco smoking, even though the Synar amendment was the first nation-level policy to set

359 an age limit on sales and distribution of tobacco products to minors, the 1964 Surgeon General's

360 report was a milestone that brought an array of subsequent changes against tobacco smoking

361 including high excise taxes, banning of tobacco advertising to youth, and banning of tobacco

362 smoking in public places (Gruber, 2001). Previous evidence with a relatively short time span did

363 not support any immediate effect of Synar on smoking prevalence in adolescents (Fichtenberg \&

364 Glantz, 2002). In this study with more than 20 years of elapsed time since Synar, we found drops

365 in tobacco cigarette smoking incidence $\sim 10$ years after the Synar amendment. Larger drops were

366 seen for young adolescents than older adolescents.

368 It is worth noting that there were increases in smoking incidence in the mid-90s in all age groups, 369 signaling robust efforts to promote smoking for the general population. Examples of such effort 370 include significant drops of price and marked increase in advertising and marketing activities in 371 the early 1990s (Chaloupka \& Wechsler, 1997; Emery, White, \& Pierce, 2001; Fichtenberg \&

372 Glantz, 2002; Gruber, 2001; Gruber \& Zinman, 1997; Holford, Meza, et al., 2014; Lum et al., 373 2009; Siegel et al., 1997; U.S. Surgeon General, 2012; WHO, 2015). Other potential influences

374 include the introduction of nicotine patch in the early 90s, which can provide a false promise for 375 easy cessation, as well as increased cannabis smoking in the mid-90s (Sugawara \& Nikaido, 376 2014) and potential secular changes in individual-level characteristics (e.g., risk-taking

377 behaviors). Despite these co-occurring changes, tobacco smoking incidence in adults 18 years of 378 age and older did not change appreciably after the first eight years post Synar, but decreased 379 among adolescents. Less clear is whether the promotion activities for tobacco smoking canceled 380 out any effect of Synar in the mid-90s. 
382 Summarizing from our findings for tobacco and alcohol, there does not seem to have been any

383 instant effect of NMDAA and Synar on the onset of drinking or smoking; it took $\sim 10$ years

384 before any observed variation at the population level (i.e., delays of onset and peaks at the legal

385 age). Nonetheless, any effect of such policies might be a long lasting one, as evidenced by the

386 declining trend of adolescent drinking and smoking that continued into the second decade after

387 NMDAA and Synar.

388

389 The results from this study may have important implications in future efforts to seek empirical

390

evidence about potential effects of policy changes on psychoactive drugs, including evidence

391 about cannabis during an era of cannabis policy change (Pacula et al., 2014). Based upon

392 findings of this study, no significant shift in the incidence curve for cannabis onset would occur

393 during the first 10 years after the implementation of cannabis policies. This may explain the

394 mixed evidence we have seen for cannabis use; that is, the mixed evidence can be a mere

395 reflection of sampling variation when there has been no real change (Azofeifa et al., 2016;

396 Cerdá, Wall, Keyes, Galea, \& Hasin, 2012; Choo et al., 2014; Harper, Strumpf, \& Kaufman,

397 2012; Hasin et al., 2015; Keyes et al., 2016; Lynne-Landsman, Livingston, \& Wagenaar, 2013;

398 Schuermeyer et al., 2014; Wall et al., 2011). Close monitoring, with special focus on the timing

399 of onsets of youth cannabis use, might provide more definitive evidence about the lag time for

400 cannabis. Nonetheless, local-level (e.g., school-based) prevention initiatives might have a more

401 immediate effect on adolescent cannabis onset, and these initiatives might well be needed during

402 the first 10 years after any nation-scale policy change in order to reduce youth cannabis onset 
403 and to prevent unnecessary adverse consequences of early onset of cannabis use, which might

404 include cognitive impairments, drugged driving, and dropping out of school (Pacula et al., 2014).

405

406

407 Acknowledgments

408 The authors are grateful to the United States Department of Health and Human Services,

409 Substance Abuse and Mental Health Services Administration for making the data publicly

410 available. Authors are grateful for valuable advice from Dr. Carla Storr.

411

\section{References}

413 Bauer, U. E., P. A. Briss, R. A. Goodman and B. A. Bowman (2014). "Prevention of chronic

414 disease in the 21 st century: elimination of the leading preventable causes of premature death and

415 disability in the USA." Lancet 384(9937): 45-52.

416 Center for Behavioral Health Statistics and Quality (2012). Comparing and evaluating youth

417 substance use estimates from the National Survey on Drug Use and Health and other surveys

418 (HHS Publication No. SMA 12-4727, Methodology Series M-9). Rockville, MD, Substance

419 Abuse and Mental Health Services Administration.

420 Dawson, D. A. (2000). "Alcohol consumption, alcohol dependence, and all-cause mortality."

421 Alcohol Clin Exp Res 24(1): 72-81.

422 Dawson, D. A., R. B. Goldstein, T. D. Saha and B. F. Grant (2015). "Changes in alcohol

423 consumption: United States, 2001-2002 to 2012-2013." Drug Alcohol Depend 148: 56-61.

424 Deandrea, D. C., J. P. Troost and J. C. Anthony (2013). "Toward primary prevention of extra-

425 medical OxyContin(R) use among young people." Prev Med 57(3): 244-246. 
426 Degenhardt, L., W. T. Chiu, N. Sampson, R. C. Kessler and J. C. Anthony (2007).

427 "Epidemiological patterns of extra-medical drug use in the United States: evidence from the

428 National Comorbidity Survey Replication, 2001-2003." Drug Alcohol Depend 90(2-3): 210-223.

429 DerSimonian, R. and N. Laird (1986). "Meta-analysis in clinical trials." Control Clin Trials 7(3):

$430 \quad 177-188$.

431 Giovannucci, E., G. Colditz, M. J. Stampfer, E. B. Rimm, L. Litin, L. Sampson and W. C.

432 Willett (1991). "The assessment of alcohol consumption by a simple self-administered

433 questionnaire." Am J Epidemiol 133(8): 810-817.

434 Grucza, R. A., K. Norberg, K. K. Bucholz and L. J. Bierut (2008). "Correspondence between secular changes in alcohol dependence and age of drinking onset among women in the United

436 States." Alcohol Clin Exp Res 32(8): 1493-1501.

437 Hasin, D. S., F. S. Stinson, E. Ogburn and B. F. Grant (2007). "Prevalence, correlates, disability, 438 and comorbidity of DSM-IV alcohol abuse and dependence in the United States: results from the 439 National Epidemiologic Survey on Alcohol and Related Conditions." Arch Gen Psychiatry 64(7): $830-842$.

441 Hermos, J. A., M. R. Winter, T. C. Heeren and R. W. Hingson (2008). "Early Age-of-onset

442 Drinking Predicts Prescription Drug Misuse Among Teenagers and Young Adults: Results from 443 a National Survey." J Addict Med 2(1): 22-30.

444 Higgins, J. P., S. G. Thompson, J. J. Deeks and D. G. Altman (2003). "Measuring inconsistency 445 in meta-analyses." BMJ 327(7414): 557-560.

446 Hingson, R. W., E. M. Edwards, T. Heeren and D. Rosenbloom (2009). "Age of drinking onset 447 and injuries, motor vehicle crashes, and physical fights after drinking and when not drinking." 448 Alcohol Clin Exp Res 33(5): 783-790. 
449 Jackson, K. M. (2010). "Progression through early drinking milestones in an adolescent 450 treatment sample." Addiction 105(3): 438-449.

451 Kalaydjian, A., J. Swendsen, W. T. Chiu, L. Dierker, L. Degenhardt, M. Glantz, K. R.

452 Merikangas, N. Sampson and R. Kessler (2009). "Sociodemographic predictors of transitions 453 across stages of alcohol use, disorders, and remission in the National Comorbidity Survey

454 Replication." Compr Psychiatry 50(4): 299-306.

455 Kanny, D., R. D. Brewer, J. B. Mesnick, L. J. Paulozzi, T. S. Naimi and H. Lu (2015). "Vital

456 signs: alcohol poisoning deaths - United States, 2010-2012." MMWR Morb Mortal Wkly Rep 457 63(53): 1238-1242.

458 Kelly, A. B., M. O'Flaherty, J. W. Toumbourou, J. P. Connor, S. A. Hemphill and R. F. Catalano 459 (2011). "Gender differences in the impact of families on alcohol use: a lagged longitudinal study 460 of early adolescents." Addiction 106(8): 1427-1436.

461 Kerr, W. C., T. K. Greenfield, J. Bond, Y. Ye and J. Rehm (2009). "Age-period-cohort 462 modelling of alcohol volume and heavy drinking days in the US National Alcohol Surveys:

463 divergence in younger and older adult trends." Addiction 104(1): 27-37.

464 Kerr, W. C., T. K. Greenfield, Y. Ye, J. Bond and J. Rehm (2013). "Are the 1976-1985 birth 465 cohorts heavier drinkers? Age-period-cohort analyses of the National Alcohol Surveys 1979466 2010." Addiction 108(6): 1038-1048.

467 Keyes, K. M., B. F. Grant and D. S. Hasin (2008). "Evidence for a closing gender gap in alcohol 468 use, abuse, and dependence in the United States population." Drug Alcohol Depend 93(1-2): 2146929.

470 Keyes, K. M., G. Li and D. S. Hasin (2011). "Birth cohort effects and gender differences in 471 alcohol epidemiology: a review and synthesis." Alcohol Clin Exp Res 35(12): 2101-2112. 
472 Keyes, K. M., S. S. Martins, C. Blanco and D. S. Hasin (2010). "Telescoping and gender

473 differences in alcohol dependence: new evidence from two national surveys." Am J Psychiatry

$474 \quad$ 167(8): 969-976.

475 Parra, G. R., S. E. O'Neill and K. J. Sher (2003). "Reliability of self-reported age of substance 476 involvement onset." Psychol Addict Behav 17(3): 211-218.

477 Penne, M. A., J. T. Lessler, G. Bieler and R. Caspar (1998). Effects of experimental audio 478 computer-assisted self-interviewing (ACASI) procedures on reported drug use in the NHSDA:

479 Results from the 1997 CAI field experiment. . 1998 Joint Statistical Meetings, American 480 Statistical Association, Social Statistics Section, Dallas, TX, American Statistical Association. . 481 Salas-Wright, C. P., M. G. Vaughn, J. Ugalde and J. Todic (2015). "Substance use and teen 482 pregnancy in the United States: evidence from the NSDUH 2002-2012." Addict Behav 45: 218 483225.

484 Sartor, C. E., K. K. Bucholz, E. C. Nelson, P. A. Madden, M. T. Lynskey and A. C. Heath 485 (2011). "Reporting bias in the association between age at first alcohol use and heavy episodic 486 drinking." Alcohol Clin Exp Res 35(8): 1418-1425.

487 Seedall, R. B. and J. C. Anthony (2015). "Monitoring by Parents and Hypothesized Male-Female 488 Differences in Evidence from a Nationally Representative Cohort Re-sampled from Age 12 to 17 489 Years: An Exploratory Study Using a "Mutoscope" Approach." Prev Sci 16(5): 696-706.

490 Shillington, A. M. and J. D. Clapp (2000). "Self-report stability of adolescent substance use: are 491 there differences for gender, ethnicity and age?" Drug Alcohol Depend 60(1): 19-27.

492 Shillington, A. M., S. I. Woodruff, J. D. Clapp, M. B. Reed and H. Lemus (2012). "Self493 Reported Age of Onset and Telescoping for Cigarettes, Alcohol, and Marijuana Across Eight 
494 Years of the National Longitudinal Survey of Youth." J Child Adolesc Subst Abuse 21(4): 333495348.

496 United States Department of Health and Human Services. Substance Abuse and Mental Health 497 Services Administration. Center for Behavioral Health Statistics and Quality (2015). National 498 Survey on Drug Use and Health: 2-Year R-DAS (2002 to 2003, 2004 to 2005, 2006 to 2007 , 4992008 to 2009, 2010 to 2011, and 2012 to 2013). ICPSR34482-v3. Ann Arbor, MI, Inter500 university Consortium for Political and Social Research.

501 Wilsnack, R. W., N. D. Vogeltanz, S. C. Wilsnack, T. R. Harris, S. Ahlstrom, S. Bondy, L. 502 Csemy, R. Ferrence, J. Ferris, J. Fleming, K. Graham, T. Greenfield, L. Guyon, E. Haavio503 Mannila, F. Kellner, R. Knibbe, L. Kubicka, M. Loukomskaia, H. Mustonen, L. Nadeau, A. 504 Narusk, R. Neve, G. Rahav, F. Spak, M. Teichman, K. Trocki, I. Webster and S. Weiss (2000). 505 "Gender differences in alcohol consumption and adverse drinking consequences: cross-cultural 506 patterns." Addiction 95(2): 251-265.

507 Wojtyniak, B., J. Moskalewicz, J. Stokwiszewski and D. Rabczenko (2005). "Gender-specific 508 mortality associated with alcohol consumption in Poland in transition." Addiction 100(12): 1779$509 \quad 1789$. 
Table $\mathbf{1}$ (on next page)

Estimated Difference in Incidence $(\%$ and $95 \% \mathrm{Cl}$ ) of Alcohol Drinking and Tobacco Cigarette Smoking Across Different Time Periods Based on the National Minimum Drinking Age Act and Synar Amendment. Data from NSDUH, 1979-2015. 
Table 1. Estimated Difference in the Incidence (\% and 95\% confidence intervals) of Alcohol Drinking and Tobacco Cigarette Smoking Across Different Time Periods Based on the National Minimum Drinking Age Act and Synar Amendment. Data from the National Survey on Drug Use and Health, 1979-2015.

\begin{tabular}{|c|c|c|c|c|c|c|}
\hline Age & $\begin{array}{c}\text { Period } 3 \text { vs. } \\
\text { Period } 2 \\
\end{array}$ & $\begin{array}{l}\text { Period } 3 \text { vs. } \\
\text { Period } 1 \\
\end{array}$ & $\begin{array}{c}\text { Period } 2 \text { vs. } \\
\text { Period } 1 \\
\end{array}$ & $\begin{array}{l}\text { Period } 3 \text { vs. } \\
\text { Period } 0 \\
\end{array}$ & $\begin{array}{l}\text { Period } 2 \text { vs. } \\
\text { Period } 0\end{array}$ & $\begin{array}{l}\text { Period } 1 \text { vs. } \\
\text { Period } 0 \\
\end{array}$ \\
\hline & \multicolumn{6}{|c|}{ Alcohol } \\
\hline 12 & $-3.6(-5.2,-2.0)$ & $-3.9(-5.8,-1.9)$ & $-0.3(-2.2,1.7)$ & $-15.9(-20.9,-10.9)$ & $-12.3(-17.3,-7.3)$ & $-12.1(-17.2,-6.9)$ \\
\hline 13 & $-4.7(-6.3,-3.1)$ & $-5.5(-7.6,-3.4)$ & $-0.8(-2.9,1.4)$ & $-19.2(-23.5,-14.8)$ & $-14.4(-18.8,-10.1)$ & $-13.7(-18.3,-9.1)$ \\
\hline 14 & $-5.0(-7.3,-2.7)$ & $-4.3(-7.0,-1.5)$ & $0.7(-2.0,3.5)$ & $-14.1(-19.9,-8.4)$ & $-9.1(-14.8,-3.4)$ & $-9.9(-15.8,-3.9)$ \\
\hline 15 & $-4.4(-6.5,-2.3)$ & $-2.1(-4.9,0.7)$ & $2.3(-0.5,5.1)$ & $-22.6(-28.8,-16.3)$ & $-18.1(-24.4,-11.9)$ & $-20.5(-27.0,-13.9)$ \\
\hline 16 & $-2.4(-4.2,-0.6)$ & $-3.4(-6.0,-0.9)$ & $-1.0(-3.6,1.6)$ & $-17.9(-24.0,-11.9)$ & $-15.5(-21.5,-9.5)$ & $-14.5(-20.8,-8.2)$ \\
\hline 17 & $-2.6(-4.5,-0.6)$ & $-1.3(-4.6,2.0)$ & $1.3(-2.1,4.6)$ & $-18.3(-26.0,-10.5)$ & $-15.7(-23.5,-8.0)$ & $-17.0(-25.2,-8.8)$ \\
\hline 18 & $1.1(-1.7,3.9)$ & $4.7(0.1,9.3)$ & $3.6(-1.0,8.2)$ & $-14.7(-25.6,-3.7)$ & $-15.8(-26.8,-4.9)$ & $-19.4(-30.9,-7.9)$ \\
\hline 19 & $-2.0(-4.7,0.6)$ & $2.1(-2.3,6.4)$ & $4.1(-0.3,8.5)$ & $-22.6(-32.6,-12.6)$ & $-20.6(-30.6,-10.6)$ & $-24.7(-35.3,-14.1)$ \\
\hline 20 & $0.4(-2.6,3.4)$ & $6.4(1.9,10.9)$ & $6.0(1.4,10.6)$ & $-12.4(-26.0,1.2)$ & $-12.8(-26.4,0.8)$ & $-18.8(-32.8,-4.8)$ \\
\hline 21 & $13.1(8.4,17.8)$ & $27.3(20.4,34.2)$ & $14.2(7.2,21.1)$ & $29.4(16.1,42.7)$ & $16.3(2.9,29.7)$ & $2.1(-12.2,16.4)$ \\
\hline \multirow[t]{2}{*}{$22-23$} & $1.8(0.6,2.9)$ & $3.8(2.3,5.2)$ & $2.0(0.6,3.4)$ & $-0.4(-5.7,5.0)$ & $-2.1(-7.5,3.2)$ & $-4.1(-9.6,1.3)$ \\
\hline & \multicolumn{6}{|c|}{ Tobacco } \\
\hline 12 & $-3.1(-4.3,-1.9)$ & $-8.0(-9.5,-6.4)$ & $-4.8(-6.4,-3.3)$ & $-8.1(-10.0,-6.1)$ & $-4.9(-6.9,-3.0)$ & $-0.1(-2.3,2.1)$ \\
\hline 13 & $-3.9(-6.3,-1.6)$ & $-9.6(-12.2,-7.0)$ & $-5.7(-8.1,-3.2)$ & $-8.2(-11.2,-5.3)$ & $-4.3(-7.1,-1.4)$ & $1.4(-1.7,4.5)$ \\
\hline 14 & $-4.1(-6.5,-1.7)$ & $-9.5(-12.2,-6.7)$ & $-5.4(-8.0,-2.8)$ & $-5.1(-8.1,-2.1)$ & $-1 \cdot 0(-3.9,1.9)$ & $4.4(1.2,7.5)$ \\
\hline 15 & $-3.7(-5.9,-1.5)$ & $-7.7(-10.3,-5.1)$ & $-4.0(-6.5,-1.5)$ & $-3.5(-6.5,-0.4)$ & $0.2(-2.7,3.1)$ & $4.2(1.0,7.5)$ \\
\hline 16 & $-3.3(-4.8,-1.8)$ & $-6.3(-8.4,-4.3)$ & $-3.0(-5.0,-1.0)$ & $-0.7(-3.0,1.6)$ & $2.6(0.3,4.8)$ & $5.6(3.0,8.2)$ \\
\hline 17 & $-0.9(-2.6,0.8)$ & $-4.5(-6.9,-2.2)$ & $-3.6(-5.9,-1.3)$ & $1.8(-1.1,4.8)$ & $2.7(-0.2,5.7)$ & $6.3(3.0,9.7)$ \\
\hline 18 & $2.4(-0.4,5.1)$ & $3.8(0.5,7.1)$ & $1.4(-1.7,4.5)$ & $12.0(8.5,15.4)$ & $9.6(6.3,12.9)$ & $8.2(4.4,11.9)$ \\
\hline 19 & $1.8(>-0.1,3.6)$ & $1.3(-1.2,3.8)$ & $-0.5(-2.9,1.9)$ & $5.1(2.0,8.1)$ & $3.3(0.3,6.2)$ & $3.8(0.3,7.2)$ \\
\hline 20 & $1.6(0.1,3.2)$ & $1.1(-1.0,3.1)$ & $-0.6(-2.4,1.3)$ & $4.1(1.6,6.6)$ & $2.4(0.0,4.8)$ & $3.0(0.3,5.7)$ \\
\hline 21 & $-0.1(-1.7,1.5)$ & $-0.7(-2.9,1.5)$ & $-0.6(-2.7,1.4)$ & $4.5(2.3,6.7)$ & $4.6(2.5,6.7)$ & $5.2(2.7,7.8)$ \\
\hline $22-23$ & $0.2(-0.2,0.7)$ & $0.6(0.1,1.1)$ & $0.3(-0.2,0.8)$ & $1.4(0.8,1.9)$ & $1.1(0.6,1.6)$ & $0.8(0.2,1.3)$ \\
\hline
\end{tabular}

For alcohol drinking, Period 0=1979-1986, Period 1=1987-1996, Period 2=1997-2006, Period 3=2007-2015; for tobacco 
Figure 1 (on next page)

Estimated Annual Incidence (\%) of Alcohol Drinking in the United States Among 12-23 Year Olds From 1979 to 2015. Data From the United States National Surveys on Drug Use and Health, 1979-2015 ( $n=358,205)$. 
Figure 1. Estimated Annual Incidence (\%) of Alcohol Drinking in the United States Among 12-23 Year Olds From 1979 to 2015. Data From the United States National Surveys on Drug Use and Health, 1979-2015 $(n=358,205)$.

A

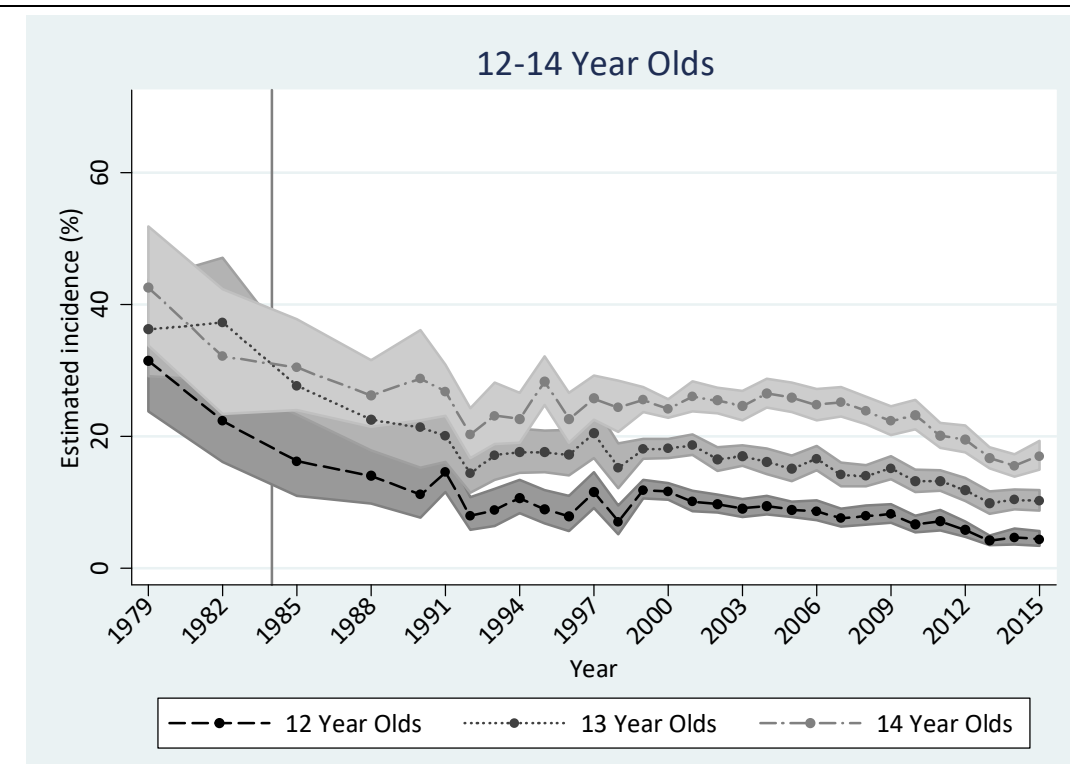

C

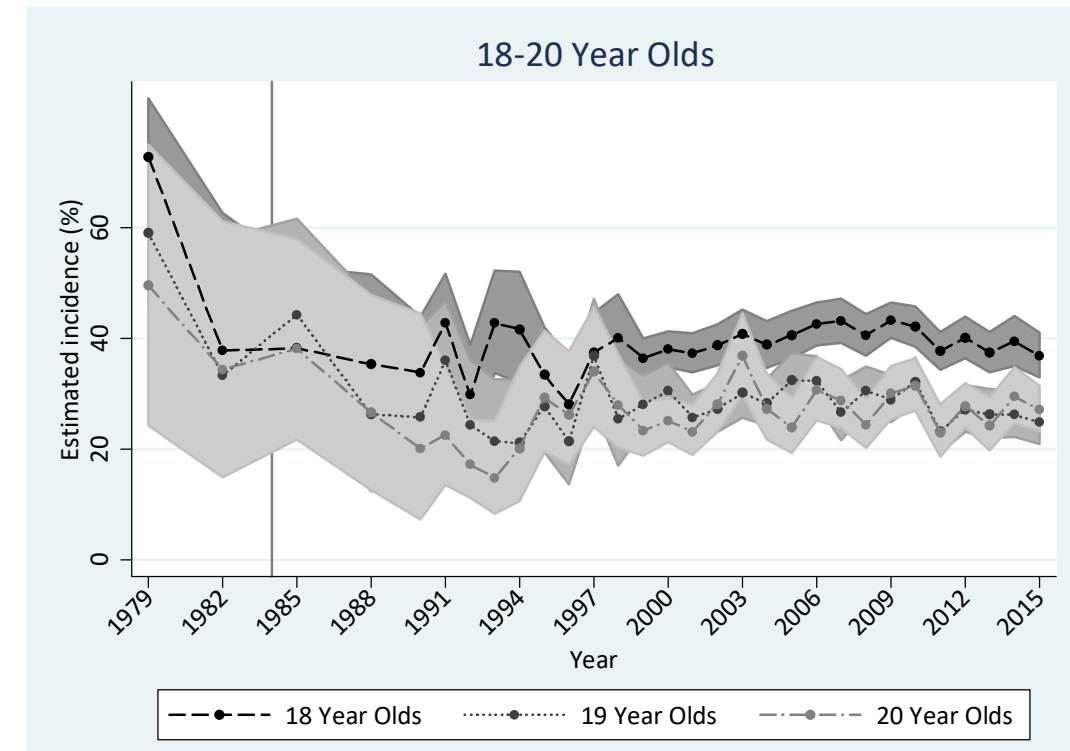

B

15-17 Year Olds

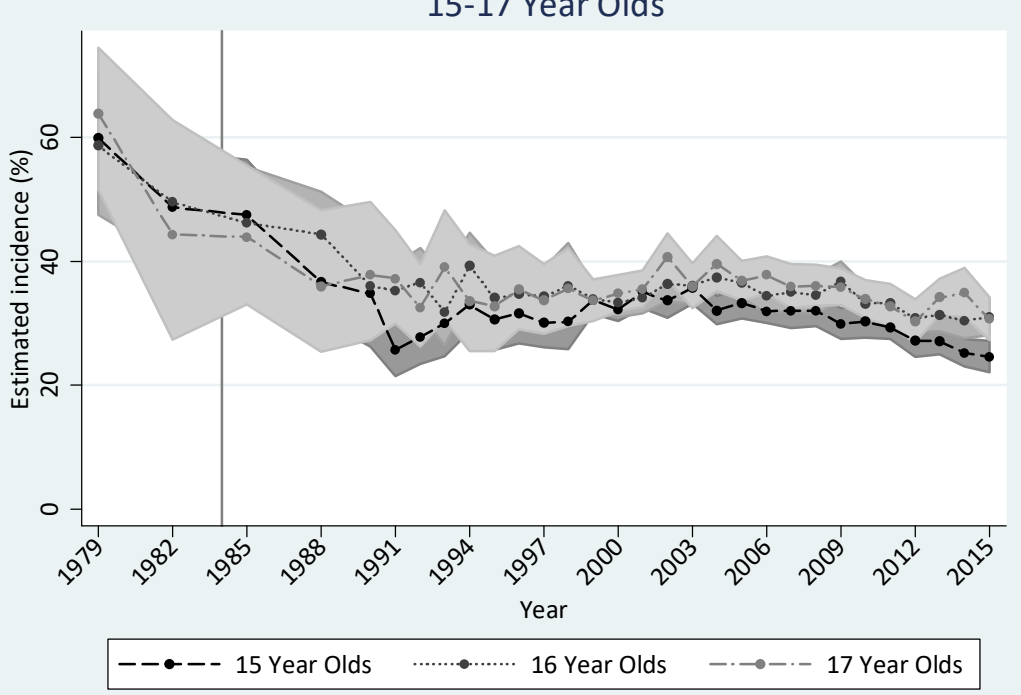

D

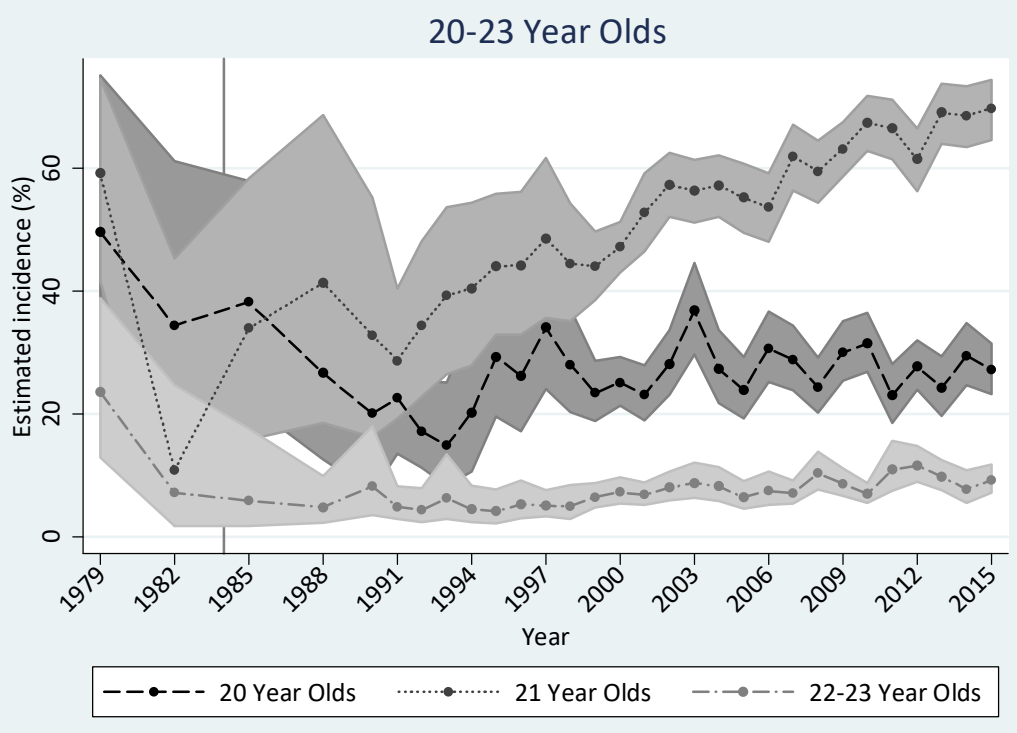

Note: Shaded areas show $95 \%$ confidence intervals. Twenty year olds appear in two plots to serve as a comparison. Vertical lines mark the year 1984, the year when NMDAA was enacted. 
Figure 2 (on next page)

Estimated Annual Incidence (\%) of Tobacco Cigarette Smoking in the United States Among 12-23 Year Olds From 1979 to 2015. Data From the United States NSDUH, 1979$2015(n=429,348)$. 
Figure 2. Estimated Annual Incidence (\%) of Tobacco Cigarette Smoking in the United States Among 12-23 Year Olds From 1979 to 2015. Data From the United States National Surveys on Drug Use and Health, 1979-2015 ( $n=429,348$ ).

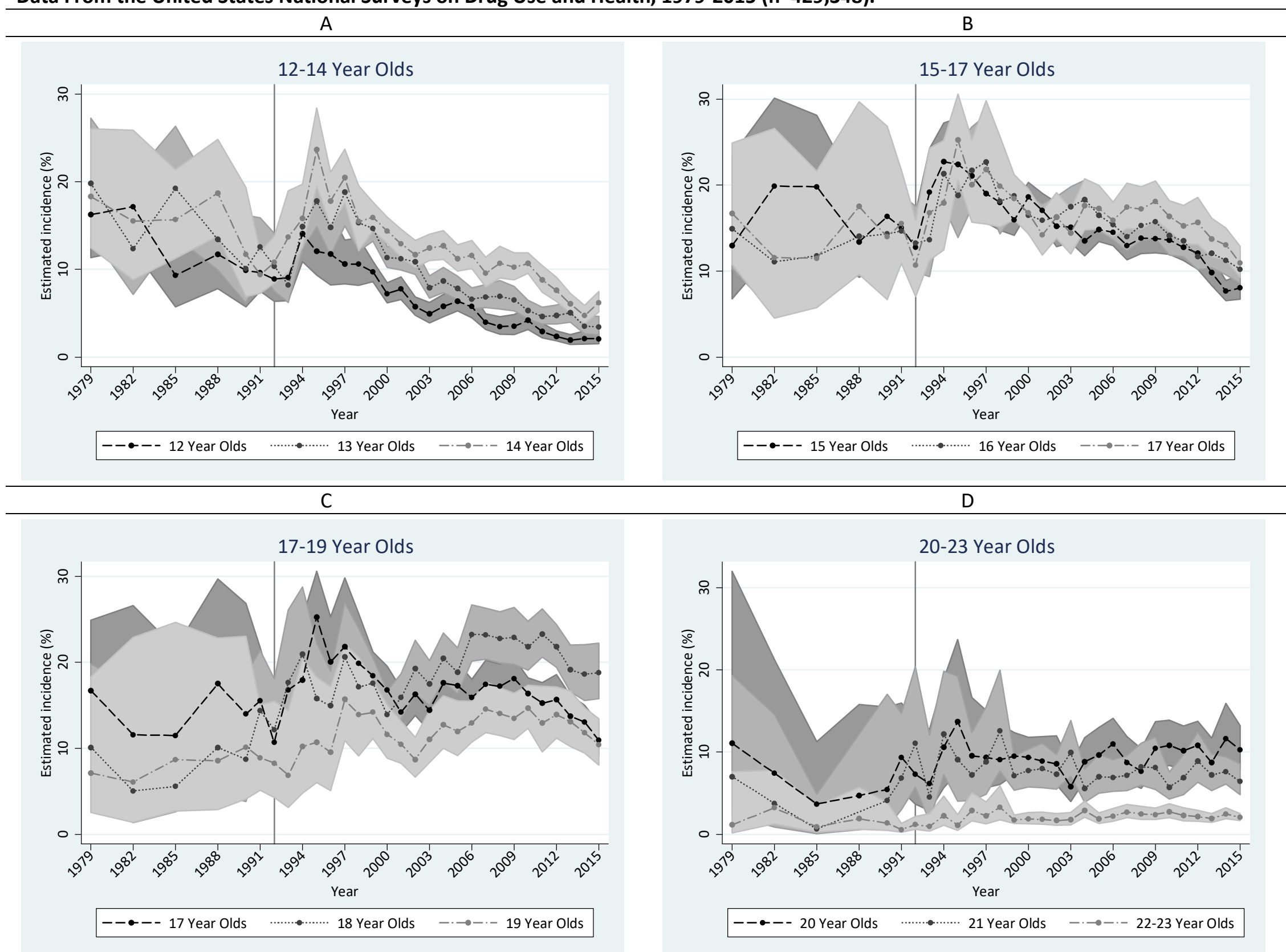

Note: Shaded areas show $95 \%$ confidence intervals. Seventeen year olds appear in two plots to serve as a comparison. Vertical lines mark the year 1992, the year when Synar was enacted. 
